\title{
Biodegradation of Tetrabromobisphenol A by strain GC under aerobic and anoxic conditions*
}

\author{
Chen Gu, Jing Wang ${ }^{\dagger}$, Ji-Ti Zhou, Hong Lu and Guang-Fei Liu \\ Key Laboratory of Industrial Ecology and Environmental Engineering \\ (Ministry of Education), School of Environmental Science and Technology, \\ Dalian University of Technology, \\ Dalian 116024, China \\ †E-mail:jwang@dlut.edu.cn \\ www.dlut.edu.cn
}

\begin{abstract}
Tetrabromobisphenol-A (TBBPA) is one of the most widely used brominates flame retardants that has extensively been detected in various environments worldwide. In the present study, the characteristics of co metabolic degradation of TBBPA by a facultative bacterium strain GC were investigated. TBBPA (10 $\left.\mathrm{mg} \mathrm{L}^{-1}\right)$ could be removed approximately $40 \%$ and $60 \%$ by strain under aerobic and anoxic conditions after 7 days cultivation. These findings are of vital importance towards building a comprehensive understanding of bacteria in degradation of TBBPA in the aquatic environments.
\end{abstract}

Keywords: Aerobic Biodegradation; Aquatic Environment; Tetrabromobisphenol A.

\section{Introduction}

Tetrabromobisphenol-A (TBBPA), one of the brominated flame retardants (BFRs), has been widely detected in the environment due to their extensively applied in the consumer products including polycarbonate plastics, textiles and electronic equipments[1]. Production of TBBPA is estimated with the annual world production may be close to 121,000 tons[2]. Although it can be covalently bound to the materials, TBBPA has been ubiquitously detected in various environmental settings and organisms[3-5]. Moreover, due to its structural similarity to thyroxin and steroid estrogen, it has been suspected that it works functionally as an endocrine disrupting chemical[6,7].

Microbial degradation is an eco-friendly process that play a major role in the removal of TBBPA from the natural environment. Most previous studies have extensively focused on anaerobic conditions since TBBPA is prone to reductively debromination yielding less brominated bisphenol A (BPA), but further anaerobic biodegradation of BPA was not observed[8-10].Previous

\footnotetext{
* This work is supported by the National Natural Science Foundations of China (21077021 and
} 51278080). 
studies have shown that the co-metabolic reaction can convert recalcitrant compounds to intermediates via catabolic and anabolic pathways of microorganisms which may be more prone to biodegradation[11].Moreover, TBBPA is long-term exposed to aerobic environments before entering the anaerobic environments, and usually co-exists with other organic contaminants. In this regard, it is crucial to understand what extent the aquatic microorganisms may contribute to the elimination of TBBPA. However, TBBPA biodegradation under aerobic conditions are limited[12-14].Thus, there is a urgent need to better understand what extent the microorganisms may contribute to the elimination of this compound in the aquatic environments.

In the present study, a bacterial strain termed strain GC was capable of degrading TBBPA under both aerobic and anoxic conditions. These results provide crucial information for unraveling biodegradation of TBBPA and can lead to the development of strategies to control TBBPA contamination in the aquatic environments.

\section{Materials and Method}

\subsection{Chemicals}

TBBPA (98\% purity) was purchased from Tokyo Chemical Industry Co., Ltd. (Tokyo, Japan). HPLC grade methanol was purchased from Tedia Company Inc. (USA). All other chemicals were reagent grade or better. Ultrapure water obtained from a Milli-Q water purification system (Millipore, USA).

\subsection{Bacteria and culture media}

Strain GC was isolated from sewage outlets of Heishijiao in Dalian, China. Strain was grown aerobically in Luria-Bertani (LB) broth. TBBPA-degrading experiments were conducted in mineral salt (MS) medium containing beef extract $(0.10 \%, w / v)$ and peptone $(0.20 \%, w / v)$. The $\mathrm{pH}$ of the media were adjusted to $8.0 \pm 0.1$ after autoclaving at $121{ }^{\circ} \mathrm{C}$ for $20 \mathrm{~min}$ before use.

\subsection{Enrichment and isolation of the TBBPA-degrading bacteria}

In brief, the enrichment was performed in $100 \mathrm{~mL}$ MS medium containing 10 $\mathrm{mg} \mathrm{L} \mathrm{L}^{-1} \mathrm{TBBPA}$ and $3 \mathrm{~g}$ (wet weight) sediment and incubated at $25^{\circ} \mathrm{C}$ in an orbital shaker operating with a speed of $150 \mathrm{rpm}$. After 7 days inoculation, a 10 $\mathrm{mL}$ aliquot of the enrichment culture was transferred to fresh MS medium containing $20 \mathrm{mg} \mathrm{L}^{-1}$ TBBPA. After five successive transfer in the same method, the concentration of TBBPA was increased to $50 \mathrm{mg} \mathrm{L}^{-1}$ to enrich the TBBPAdegrading bacteria. The cultures capable of degrading TBBPA were repetitive 
spread on solidified MS medium (1.5\% agar) and supplemented with $50 \mathrm{mg} \mathrm{L}^{-1}$ TBBPA to obtain pure cultures. Only one type of colony was obtained.

\subsection{Effects of substrate, $\mathrm{pH}$, and initial inoculation on aerobic biodegradation of TBBPA}

To investigate the effect of substrate concentration on biodegradation of TBBPA (10 $\mathrm{mg} \mathrm{L}^{-1}$ ), different concentrations of beef extract and peptone were carried out. To investigate the effect of $\mathrm{pH}$ on biodegradation of TBBPA $\left(10 \mathrm{mg} \mathrm{L}^{-1}\right)$, different $\mathrm{pH}$ values $(7,8,9,10,11)$ of substrate were conducted. To investigate the effect of initial inoculation on biodegradation of TBBPA $\left(10 \mathrm{mg} \mathrm{L}^{-1}\right)$, different initial inoculations (1-10\%) were conducted. To avoid the photolysis of TBBPA, all the experiments were conducted in the dark.

\subsection{Aerobic and anoxic biodegradation of TBBPA}

To compare the aerobic and anoxic biodegradation of TBBPA, we conducted the biodegradation of TBBPA under aerobic and anoxic cultivations in Erlenmeyer flasks and serum bottles at $25^{\circ} \mathrm{C}$, respectively. TBBPA $\left(10 \mathrm{mg} \mathrm{L}^{-1}\right)$ was added to the flasks and bottles containing MS medium, respectively. Control experiments without strain were also conducted. For aerobic experiments, flasks with cotton-wool stoppers were used to allow air exchange. For anoxic experiments, bottles were sealed with butyl rubber stoppers and aluminum crimp seals.

\subsection{Analytical methods}

Samples were sacrificed to determine the residual concentration of TBBPA through $\mathrm{Ph}$ adjustment to above 9.0 and balanced for $30 \mathrm{~min}$. TBBPA was detected using high performance liquid chromatography (HPLC, Shimadzu LC20AT) with a diode array detector at $290 \mathrm{~nm}$. The mobile phase consisted of methanol (80\%) and $1.0 \%$ acetic acid (20\%) at a flow rate of $1 \mathrm{~mL} \mathrm{~min}^{-1}[15]$.

\section{Results and Discussion}

\subsection{Isolation of the TBBPA-degrading bacterium}

After five successive transfer, the various colonies were picked and isolated as pure cultures. The strain was purified by transferring the colonies several times onto fresh agar plates. One yellow type of colony was obtained and termed strain GC for subsequent assays. 


\subsection{Effects of substrate, $p H$, and inoculation on biodegradation of TBBPA}

Figure 1 shows that the biodegradation efficiency of TBBPA was approximately $40 \%$ after 7 days cultivation in the presence of beef extract $(0.10 \%, w / v)$ and peptone $(0.20 \%, w / v)$, indicating that beef extract and peptone could provide important nutrients for the growth of strain. Results further showed that the optimal $\mathrm{pH}$ values of MS medium on TBBPA degradation was nearly the same (Fig. 2). To simulate the natural estuary environments, we selected the $\mathrm{pH}$ of 8.0 for subsequent assays. The optimal initial inoculation on TBBPA degradation was $5 \%$ (Fig. 3). Taken together, TBBPA could be removed approximately $40 \%$ by strain after 7 days cultivation under optimized conditions (MS medium, $\mathrm{pH}$ 8.0 , inoculation $5 \%$ ).

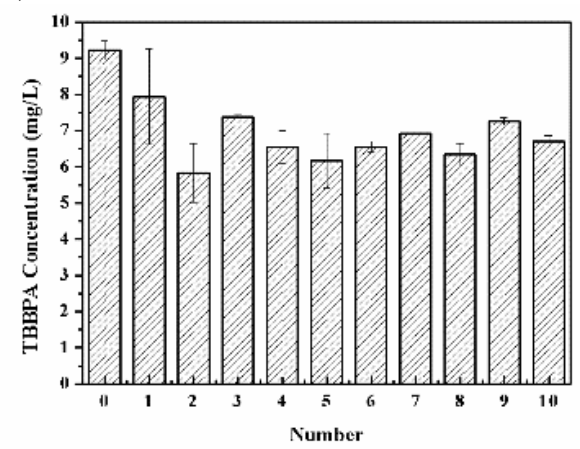

Fig. 1. Effect of substrate concentration on biodegradation of TBBPA $(1,0.05 \% \mathrm{BE}+0.10 \% \mathrm{P} ; 2$, $0.10 \% \mathrm{BE}+0.20 \% \mathrm{P} ; 3,0.15 \% \mathrm{BE}+0.30 \% \mathrm{P} ; 4,0.20 \% \mathrm{BE}+0.40 \% \mathrm{P} ; 5,0.25 \% \mathrm{BE}+0.50 \% \mathrm{P} ; 6$, $0.30 \% \mathrm{BE}+0.60 \% \mathrm{P} ; 7,0.35 \% \mathrm{BE}+0.60 \% \mathrm{P} ; 8,0.40 \% \mathrm{BE}+0.80 \% \mathrm{P} ; 9,0.45 \% \mathrm{BE}+0.90 \% \mathrm{P} ; 10$, $0.50 \% \mathrm{BE}+1.00 \% \mathrm{P} ; 0$, control-10).

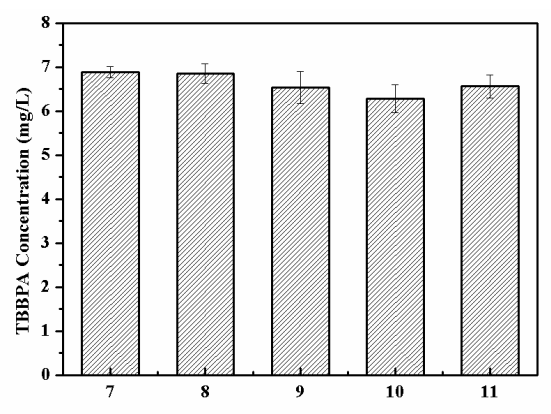

Fig. 2. Effect of $\mathrm{pH}$ on biodegradation of TBBPA. 


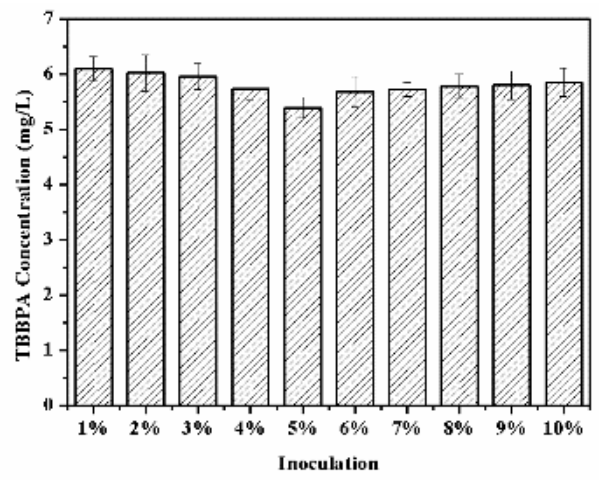

Fig. 3. Effect of inoculation on biodegradation of TBBPA.

\subsection{Aerobic and anoxic biodegradation of TBBPA}

Figure 4 shows that anoxic biodegradation of TBBPA was higher than aerobic biodegradation within 7 days cultivation. Indeed, most previous studies have extensively focused on anaerobic conditions since TBBPA is prone to reductively debromination yielding less brominated BPA, but further anaerobic biodegradation of BPA was not observed. In the present study, after 7 days cultivation, the degradation efficiencies of TBBPA were approximately $40 \%$ and $60 \%$ under aerobic and anoxic conditions, respectively. In addition, TBBPA as the sole carbon source could not be degraded by strain (data not shown), suggesting that TBBPA could not be used as primary substrate by strain. Thus, TBBPA was degraded via co-metabolic reaction.

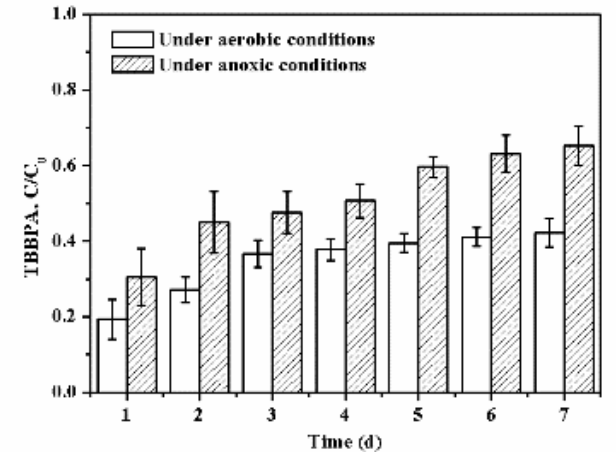

Fig. 4. Aerobic and anoxic biodegradation of TBBPA.

\section{Conclusion}

In the present study, a facultative bacterium, GC, was isolated that effectively degraded TBBPA. Our findings demonstrate that degradation of TBBPA occurs under co-metabolic condition by strain GC. Therefore we assume that this study 
could be successful in removal of TBBPA environmental burden under both aerobic and anoxic conditions. Our next goal is to characterize the detailed mechanism of direct aerobic degradation of TBBPA.

\section{References}

1. M.Alaee, et al., An overview of commercially used brominated flame retardants, their applications, their use patterns in different countries/regions and possible modes of release, Environ. Int. 29, 683-689 (2003).

2. C. A. de Wit, An overview of brominated flame retardants in the environment, Chemosphere 46, 583-624 (2002).

3. M. S. Mäkinen, et al., Respiratory and dermal exposure to organ phosphorus flame retardants and tetrabromobisphenol A at five work environments, Environ. Sci. Technol. 43, 941-947 (2009).

4. S. Morris, et al., Distribution and fate of HBCD and TBBPA brominated flame retardants in North Sea estuaries and aquatic food webs, Environ. Sci. Technol. 38, 5497-5504 (2004).

5. M. J. He, et al., Tetrabromobisphenol-A and hexabromocyclododecane in birds from an e-waste region in South China: Influence of diet on diastereoisomer-and enantiomer-specific distribution and trophodynamics, Environ. Sci. Technol. 44, 5748-5754 (2010).

6. S. Decherf, et al., Disruption of thyroid hormone-dependent hypothalamic set-points by environmental contaminants, Molecul. Cell. Endocrinol. 323, 172-182 (2010).

7. S. Kitamura, et al., Thyroid hormonal activity of the flame retardants tetrabromobisphenol A and tetrachlorobisphenol A, Biophys. Res. Commun. 293, 554-559 (2002).

8. Z. Ronen and A. Abeliovich, Anaerobic-aerobic process for microbial degradation of tetrabromobisphenol A, Appl. Environ. Microbiol. 66, 2372-2377(2000).

9. J. W.Voordeckers, et al., Anaerobic biotransformation of tetrabromobisphenol A, tetrachlorobisphenol $\mathrm{A}$, and bisphenol $\mathrm{A}$ in estuarine sediments,Environ. Sci. Technol.36,696-701(2002).

10. X.Peng, et al., Biodegradation of tetrabromobisphenol A by a novel Comamonas sp. strain, JXS-2-02, isolated from anaerobic sludge,Bioresour. Technol. 128,173-179(2013).

11. T. Yi and W.F. Harper, The link between nitrification and biotransformation of 17a-ethinylestradiol, Environ. Sci. Technol. 41, 4311-4316 (2007).

12. K. W. George and M. M. Häggblom, Microbial O-methylation of the flame retardant tetrabromobisphenol-A, Environ. Sci. Technol. 42, 5555-5561 (2008). 
13. F. Q. Peng, et al., Biotransformation of the flame retardant tetrabromobisphenol-A (TBBPA) by freshwater microalgae, Environ. Toxicol. Chem. 33,1705-1711 (2014).

14. T. An, et al., One-step process for debromination and aerobic mineralization of tetrabromobisphenol-A by a novel Ochrobactrum sp. T isolated from an e-waste recycling site,Bioresour. Technol.102,9148-9154(2011).

15. J. Wang, et al., Mediators-assisted reductive biotransformation of tetrabromobisphenol-A by Shewanella sp. XB, Bioresour. Technol. 142,192-197 (2013). 